\title{
Methanethiol as a catabolite of methionine provides methylthio- group for chemical formation of 19-S-methylgeldanamycin and 17,19-dimethylthioherbimycin A
}

\author{
Shufen $\mathrm{Li}^{1,3}$, Jing Cui ${ }^{1,3}$, Xinhua $\mathrm{Lu}^{2}$, Zhihui Zheng ${ }^{2}$, Xin $\mathrm{Liu}^{1}$, Siyang $\mathrm{Ni}^{1}$, Yiguang Wang ${ }^{1}$ and Linzhuan $\mathrm{Wu}^{1}$ \\ The Journal of Antibiotics (2013) 66, 499-503; doi:10.1038/ja.2013.31; published online 17 April 2013
}

Keywords: 17,19-dimethylthioherbimycin A; methanethiol; methionine; 19-S-methylgeldanamycin; Streptomyces hygroscopicus

Geldanamycin (GDM) is an ansamycin produced by Streptomyces hygroscopicus. In our study of secondary metabolites from S. hygroscopicus 17997 (a GDM producing strain deposited at China Pharmaceutical Culture Collection, with an accession number CPCC 200120), we identified a natural GDM analog, 19-S-methylgeldanamycin (1, Figure 1). Compound 1 retained potent cytotoxicity against cancer cells, and meanwhile exhibited increased water solubility and photostability compared with GDM. ${ }^{1}$

As a methylthio-derivative of GDM, 1's synthetic mechanism in S. hygroscopicus 17997 remains unclear. The biosynthetic pathway of GDM does not contain any methylthiolation reaction. ${ }^{2-4}$ Besides, bioinformatics analysis of GDM biosynthetic gene clusters revealed no candidate gene encoding radical-SAM enzyme for methylthiolation reaction. ${ }^{5}$ So, $19-$ methylthiolation of GDM is not an essential and/or extended modification reaction in GDM biosynthesis.

An examination of natural ansamycins reveals quite a few compounds with methylthio- group (such as trierixin, quinotrierixin, awamycin and 3-methylthiorifamycin SV, Figure 1) linking to an aromatic carbon of the molecules. ${ }^{6-9}$ Besides, urdamycin $\mathrm{E}$ as an angucycline antibiotic contains also a methylthio- group (Figure 1). ${ }^{10}$

Among these compounds, urdamycin $\mathrm{E}$ and 3-methylthiorifamycin SV were known to be closely related to L-methionine (Met) for their production. ${ }^{8,10}$ Rohr $^{10}$ proposed a mechanism for chemical conversion of urdamycin A to E, which involved a Michael addition of ${ }^{-S M e}$ (derived biogenetically from Met) to urdamycin A. The mechanism may be applicable to methylthio-containing quinone antibiotics such as awamycin. ${ }^{10}$ In fact, methanethiol can react chemically with GDM (or naphthomycin A), yielding $\mathbf{1}$ (or 30-methylthionaphthomycin A by addition-elimination). ${ }^{11,12}$
Therefore, the methylthio- group in $\mathbf{1}$ should come from methanethiol, and methanethiol should be derived from Met as a catabolite by $S$. hygroscopicus 17997. Other natural ansamycins carrying methylthio-groups (Figure 1) must have the same biological origin and synthetic mechanism.

To demonstrate the above deduction, we supplemented Met into ISP2 medium ( $1.0 \%$ malt extract, $0.4 \%$ yeast extract, $0.4 \%$ glucose, $1.5 \%$ agar, $0.3 \% \mathrm{Met}$ ) to culture S. hygroscopicus 17997 at $28^{\circ} \mathrm{C}$ for 5 days. We found that the production of 1 increased to a level of $\sim 20 \mathrm{mgl}^{-1}$ (Compound $\mathbf{1}$ was only MS detectable if Met was not supplemented). Besides, a strong and repulsive smell filled the room for culturing S. hygroscopicus 17997. GC-MS analysis confirmed that the bad smell originated from methanethiol diffused out of the headspace gas of these Met-plus ISP2 medium plates with Streptomyces hygroscopicus 17997 (Figure 2). And methanethiol as a catabolite of Met by S. hygroscopicus 17997 reacted chemically with GDM, which yielded 1 (Figure 3 and Supplementary Figure S1).

When we supplemented L-ethionine to ISP2 medium plates inoculated with S. hygroscopicus 17997, we detected the production of an expected red compound with $m / z$ 643, corresponding to the sodium adduct ion of 19-S-ethylgeldanamycin $\left(\mathrm{C}_{31} \mathrm{H}_{44} \mathrm{~N}_{2} \mathrm{O}_{9} \mathrm{SNa}\right.$; see Supplementary Information for its silica gel thin layer chromatography (TLC) and MS-MS confirmation; Supplementary Figures S2 and S3). These S. hygroscopicus 17997 cultures also gave off a strong smell of thiols (ethanethiol). This result provided an additional evidence that the methylthio- group in $\mathbf{1}$ originated from Met, which was catabolized by S. hygroscopicus 17997 to methanethiol as substrate for the production of 1 .

\footnotetext{
${ }^{1}$ Key Laboratory of Biotechnology of Antibiotics, Ministry of Health, Institute of Medicinal Biotechnology, CAMS \& PUMC, Beijing, China and ${ }^{2}$ NCPC New Drug Research and Development Co. Ltd, Shijiazhuang, Hebei, China

${ }^{3}$ These authors contribute equally to this work.

Correspondence: Professor L Wu, Key Laboratory of Biotechnology of Antibiotics, Ministry of Health, Institute of Medicinal Biotechnology, Peking Union Medical College and Chinese Academy Medical Sciences, Tiantan Xili, Beijing 100050, China.

E-mail: wulinzhuan@yahoo.com.cn or wulinzhuan@imb.pumc.edu.cn

Received 13 July 2012; revised 15 October 2012; accepted 20 March 2013; published online 17 April 2013
} 


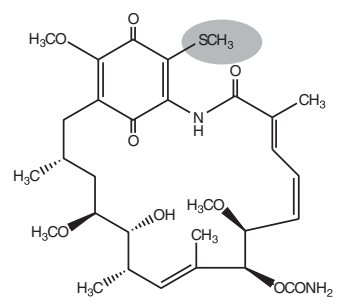

19-S-Methylgeldanamycin (1)

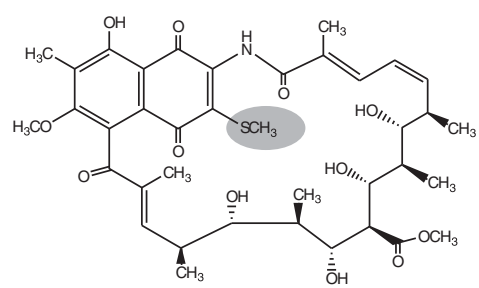

Awamycin<smiles>CO[C@H](/C=C/C=C/C=C/C[C@H](OC(=O)C(C)NC(=O)C1CCCCC1)[C@@H](C)[C@@H](O)/C(C)=C\CCc1cc(O)c([18O])c(NC(=O)CC(C)O)c1O)CO</smiles>

Trierixin

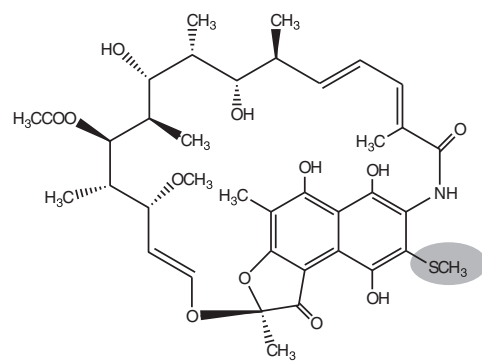

3-Methylthiorifamycin SV

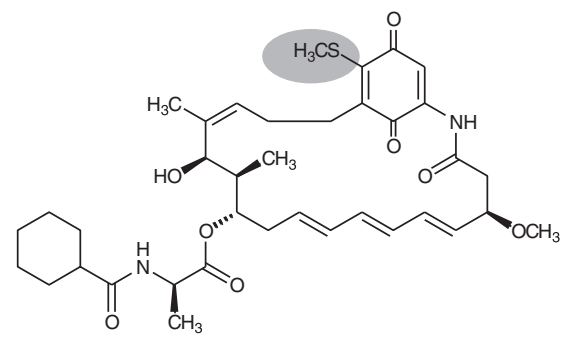

Quinotrierixin

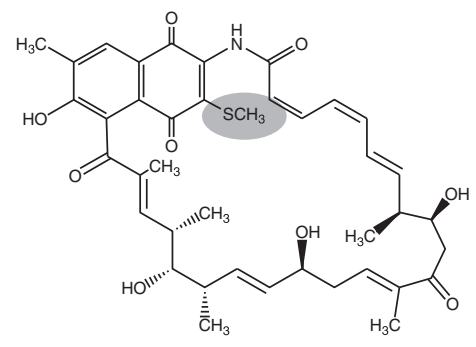

Naphthoquinomycin B

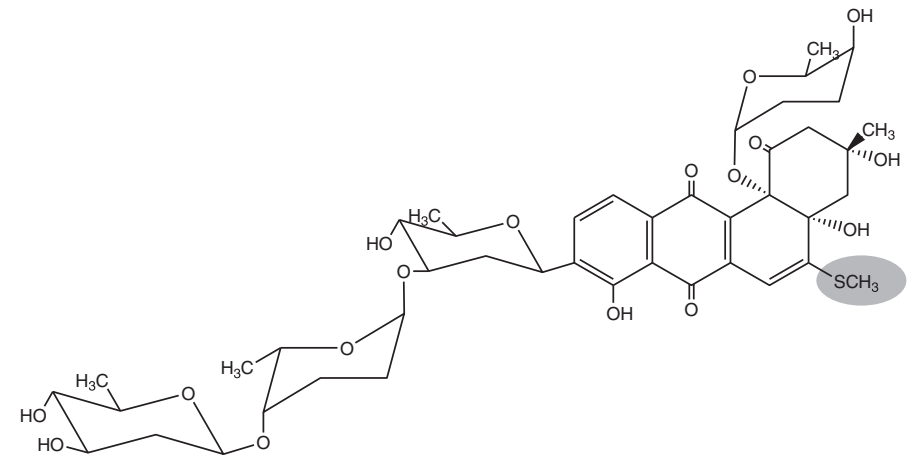

Urdamycin E

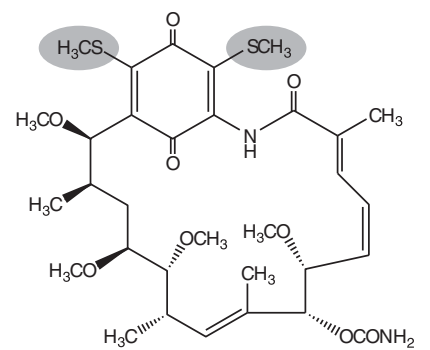

17,19-Dimethylthioherbimycin A (2)

Figure 1 Chemical structures of some natural products carrying methylthio-group. A full color version of this figure is available at The Journal of Antibiotics journal online.

To further confirm the above synthetic mechanism, we supplemented Met $\left(3.0 \mathrm{gl}^{-1}\right)$ in culturing S. hygroscopicus N02Z-0421 (a herbimycin producer isolated by China NCPC New Drug Research and Development Co. Ltd.) to obtain methylthio- derivative of herbimycin, which is a close analog of GDM. ${ }^{13}$ An expected red compound was detected in the secondary metabolites of S. hygroscopicus N02Z-0421 by silica gel TLC (Figure 4). The red compound was then purified by a procedure of ethyl acetate extraction, silica gel chromatography and reversed-phase HPLC, and $10 \mathrm{mg}$ pure preparation of the red compound (purity $>98 \%$, calculated by area\% of HPLC at $250 \mathrm{~nm}$; Supplementary Figure S4) were obtained from 1.21 fermentation supernatant of S. hygroscopicus N02Z-0421. (See Supplementary Information for detailed descriptions of fermentation of S. hygroscopicus N02Z-0421 and purification of the red compound.)

HR-ESI $(+)$-MS of the red compound revealed a principal peak at $\mathrm{m} / \mathrm{z} 689.25554\left([\mathrm{M}+\mathrm{Na}]^{+}\right)$and a minor $\left({ }^{34} \mathrm{~S}\right.$ isotope $)$ peak at $\mathrm{m} / \mathrm{z}$ 691.24797 (Supplementary Information, Supplementary Figure S5). The molecular formula of the red compound was thus established as
$\mathrm{C}_{32} \mathrm{H}_{46} \mathrm{~N}_{2} \mathrm{O}_{9} \mathrm{~S}_{2}$ (exact mass 689.25369 for $[\mathrm{M}+\mathrm{Na}]^{+}$), which is $\mathrm{S}_{2} \mathrm{C}_{2} \mathrm{H}_{4}$ (or two $\mathrm{SCH}_{2}$ ) more than herbimycin $\mathrm{A}\left(\mathrm{C}_{30} \mathrm{H}_{42} \mathrm{~N}_{2} \mathrm{O}_{9}\right)$.

The NMR spectra of the red compound (Supplementary Figures S6-S11) were highly similar to those of herbimycin A except a few differences. ${ }^{14,15}$ In the ${ }^{13} \mathrm{C}-\mathrm{NMR}$, two additional carbon signals of $-\mathrm{SCH}_{3}$ at $\delta_{\mathrm{C}} 15.1$ and $\delta_{\mathrm{C}} 16.3$ showed up; in the ${ }^{1} \mathrm{H}-\mathrm{NMR}$, two additional hydrogen signals $\left(\delta_{\mathrm{H}} 2.52, \delta_{\mathrm{H}} 2.60\right)$ from two $-\mathrm{SCH}_{3}$ appeared, whereas the hydrogen signals of C-17 and C-19 in herbimycin A disappeared. Long-range correlations from methyl proton at $\delta_{\mathrm{H}} 4.28(\mathrm{H}-15)$ and $-\mathrm{SCH}_{3}$ proton at $\delta 2.52$ to aromatic carbon $\mathrm{C}-17$ suggested that this $-\mathrm{SCH}_{3}\left(\delta_{\mathrm{H}} 2.52\right)$ group was connected to $\mathrm{C}-17$. The other $-\mathrm{SCH}_{3}$ group was connected to $\mathrm{C}-19$, as indicated by its methyl proton at $\delta_{\mathrm{H}} 2.60$ exhibiting long-range correlation to the aromatic carbon C-19. Therefore, the chemical structure of the red compound was 17,19-dimethylthioherbimycin A (2). Its NMR chemical shifts were assigned completely by HSQC, COSY and HMBC (Table 1 and Figure 5).

A comparison of the benzoquinone moiety of GDM and herbimycin A shows that C-17 of GDM links to a methoxyl group, while 

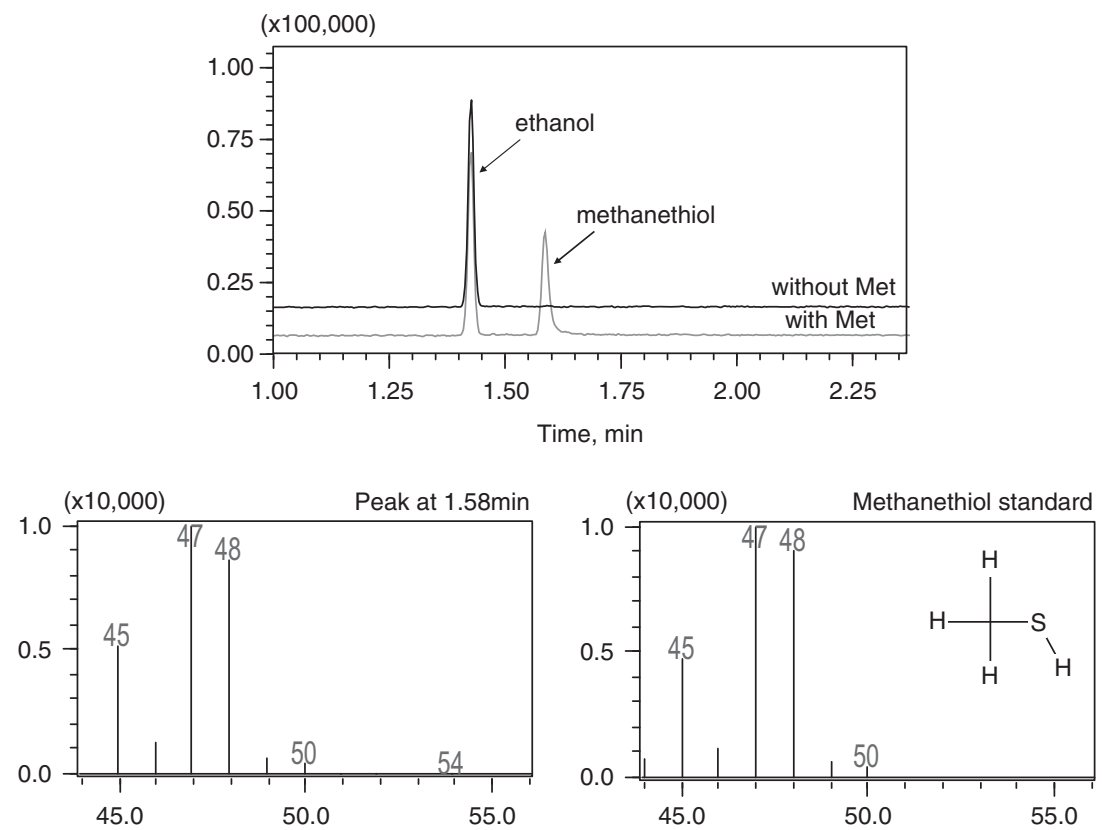

Figure 2 GC-MS of headspace gas of ISP2 medium (with and without supplementing Met) plates cultured with Streptomyces hygroscopicus 17997 at $28^{\circ} \mathrm{C}$ for 5 days. The GC-MS was performed on an Agilent QP2010 with a column Rxi-5MS $(30 \mathrm{~m} \times 0.25 \mathrm{~mm})$ under the following parameters. For GC, column oven temperature $35^{\circ} \mathrm{C}$, injection temperature $200^{\circ} \mathrm{C}$, flow control mode linear velocity $\left(36.0 \mathrm{~cm} \mathrm{~s}^{-1}\right)$, helium as carrier gas at flow rate $1.0 \mathrm{ml} \mathrm{min}{ }^{-1}$ and pressure $47.6 \mathrm{kPa}$, split ratio 100 . For MS, ion source temperature $200{ }^{\circ} \mathrm{C}$, interface temperature $250^{\circ} \mathrm{C}$, acquisition mode scan ( 285 per second), time $1.0-5.0 \mathrm{~min}, \mathrm{~m} / \mathrm{z}$ 45-100. Samples $(10 \mu \mathrm{l})$ were injected manually in split mode at $100: 1$, with injector temperature $200^{\circ} \mathrm{C}$. GC (upper): a methanethiol peak at 1.58 min appeared only from the headspace gas of culture medium with Met $\left(3.0 \mathrm{gl}^{-1}\right)$. The peak was proved to be methanethiol by MS. MS (lower): left, MS spectrum of the peak at $1.58 \mathrm{~min}$; right, standard MS spectrum of methanethiol from US National Institute of Standards and Technology. The two MS spectra were identical $\left(\mathrm{m} / \mathrm{z} 48, \mathrm{M}^{+}\right.$, molecular ion; $\left.\mathrm{m} / \mathrm{z} 47, \mathrm{M}+-\mathrm{H} ; \mathrm{m} / \mathrm{z} 45, \mathrm{M}^{+}-\mathrm{H}-2 \mathrm{H}\right)$. A full color version of this figure is available at The Journal of Antibiotics journal online.

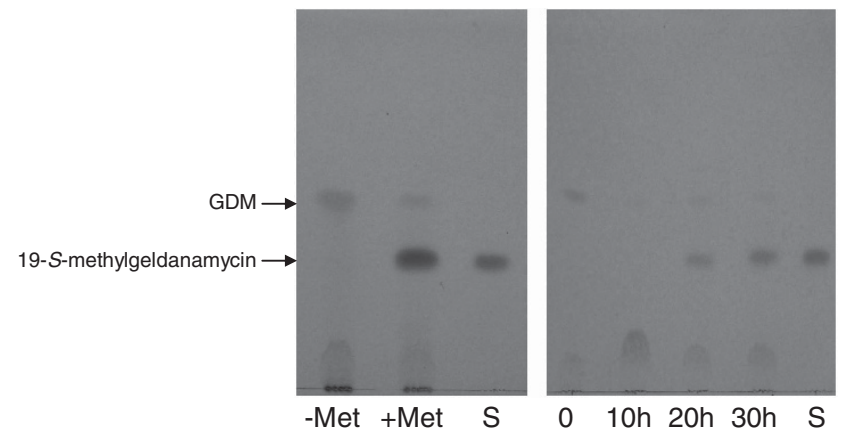

Figure 3 Silical gel TLC for 19-S-methylgeldanamycin production. Two ISP2 plates (one with $3.0 \mathrm{gl}^{-1} \mathrm{~L}$-Met, the other without L-Met as control) inoculated with Streptomyces hygroscopicus 17997 were incubated at $28^{\circ} \mathrm{C}$ for 3 days for mycelia growth. The third ISP2 plate was prepared without inoculating Streptomyces hygroscopicus 17997, but added Geldanamycin (GDM) (purity $\geqslant 95 \%$, prepared by the author's laboratory) to a final concentration of $50 \mathrm{mgl}^{-1}$. Upon removing the lids, the third ISP2 plate was placed upside down over the former one ISP2 plate with L-Met, and then incubated at $28^{\circ} \mathrm{C}$ for $0,10,20$ and $30 \mathrm{~h}$ to detect 19-S-methylgeldanamycin production. At each time, 1/4 agar culture of each plate was cut off for ethyl acetate extraction. The organic extract mixed with a small volume of $10 \% \mathrm{FeCl} 3$ for $30 \mathrm{~min}$ at room temperature, to oxidize hydroquinones to quinones, before silica gel TLC with a mobile phase of EtOAc/CH2Cl2/hexane/methanol (9:6:6:1, $/ / v)$, in which GDM had a Rf of 0.55 and $19-S$-methylgeldanamycin 0.38. The significant increase of 19-S-methylgeldanamycin production indicated that 19-S-methylgeldanamycin came from GDM reacting chemically with methanethiol. Left: 19-Smethylgeldanamycin production in the two ISP2 plates with Streptomyces hygroscopicus 17997. Right: 19-S-methylgeldanamycin production in the ISP2 plate without Streptomyces hygroscopicus 17997 (cell-free plate). S stands for $19-S$-methylgeldanamycin standard (purity $\geqslant 93 \%$, prepared by the author's laboratory). A full color version of this figure is available at The Journal of Antibiotics journal online.

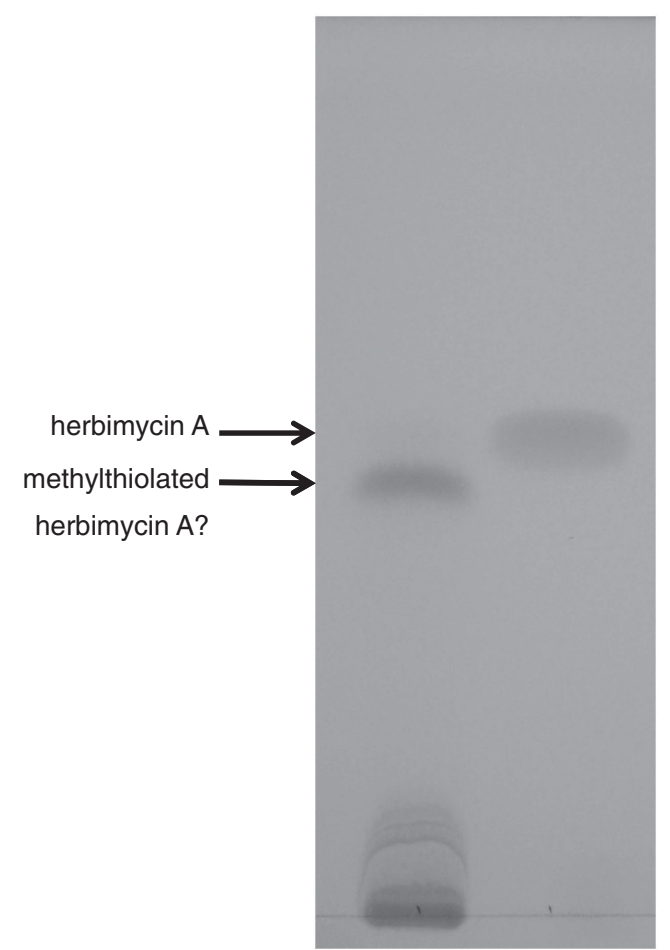

Figure 4 Silica gel TLC (developed with the same mobile phase as that of Figure 3) of ethyl acetate extract of fermentation supernatant of Streptomyces hygroscopicus N02Z-0421. Herbimycin A showed a $R_{\mathrm{f}}$ value of 0.60 , and its methylthiolated derivative 0.55 . A full color version of this figure is available at The Journal of Antibiotics journal online. 
Table 1 NMR data of 17,19-dimethylthioherbimycin A

\begin{tabular}{|c|c|c|}
\hline Position & $\delta_{C}$ & $\delta_{H}(\mathrm{~J}$ in $\mathrm{Hz})$ \\
\hline 1 & 174.5 & - \\
\hline 2 & 138.2 & - \\
\hline 3 & 123.8 & 6.38 , overlap \\
\hline 4 & 127.5 & 6.34 , overlap \\
\hline 5 & 131.7 & $5.28, \mathrm{t}(10.2)$ \\
\hline 6 & 75.9 & $4.06, \mathrm{dd}(10.2,9.0)$ \\
\hline 7 & 81.0 & $4.90, \mathrm{~d}(9.0)$ \\
\hline 8 & 128.7 & - \\
\hline 9 & 134.3 & $5.18, \mathrm{~d}(10.8)$ \\
\hline 10 & 35.5 & 2.34, brs \\
\hline 11 & 86.9 & 3.13 , overlap \\
\hline 12 & 82.2 & 3.13 , overlap \\
\hline 13 & 30.0 & $\begin{array}{c}\text { 0.93, overlap } \\
0.91 \text {, brs }\end{array}$ \\
\hline 14 & 32.7 & 2.94, brs \\
\hline 15 & 82.5 & $4.28, d(9.0)$ \\
\hline 16 & 139.7 & - \\
\hline 17 & 150.4 & - \\
\hline 18 & 178.8 & - \\
\hline 19 & 135.2 & - \\
\hline 20 & 138.4 & - \\
\hline 21 & 176.9 & - \\
\hline $2-\mathrm{CH}_{3}$ & 12.6 & $1.91, \mathrm{~s}$ \\
\hline $6-\mathrm{OCH}_{3}$ & 55.8 & $3.12, \mathrm{~s}$ \\
\hline 7-OCONH & 157.7 & - \\
\hline $8-\mathrm{CH}_{3}$ & 11.9 & $1.48, \mathrm{~s}$ \\
\hline $10-\mathrm{CH}_{3}$ & 16.4 & $0.93, d(6.6)$ \\
\hline $11-\mathrm{OCH}_{3}$ & 60.8 & $3.44, \mathrm{~s}$ \\
\hline $12-\mathrm{OCH}_{3}$ & 55.6 & $3.27, \mathrm{~s}$ \\
\hline $14-\mathrm{CH}_{3}$ & 17.0 & 1.07, d (6.6) \\
\hline $15-\mathrm{OCH}_{3}$ & 56.1 & $3.07, \mathrm{~s}$ \\
\hline $17-\mathrm{SCH}_{3}$ & 15.1 & 2.52 \\
\hline $19-\mathrm{SCH}_{3}$ & 16.3 & 2.60 \\
\hline
\end{tabular}

${ }^{1} \mathrm{H}$ - and ${ }^{13} \mathrm{C}$-NMR spectra were obtained on a Varian VNS-600 $\left({ }^{1} \mathrm{H}-\mathrm{NMR}\right.$ at $600 \mathrm{MHz},{ }^{13} \mathrm{C}-\mathrm{NMR}$ at $150 \mathrm{MHz}$ ) with $\mathrm{TMS}$ as internal standard, and measured in $\mathrm{CD}_{3} \mathrm{OD}$ at room temperature.

C-17 of herbimycin A links to a hydrogen atom that can be easily substituted by other groups. Accordingly, GDM can be monomethylthiolated at C-19, while herbimycin A di-methylthiolated at C-17,19. But it is interesting to note that both trierixin and quinotrierixin (Figure 1) are mono-methylthiolated derivatives of ansatrienin/mycotrienin, ${ }^{16,17}$ although ansatrienin contains two aromatic carbons for methylthiolation like herbimycin A.

Ansamitocin is a benzenic ansamycin produced by Actinosynnema pretiosum ATCC $31565 .{ }^{18}$ When supplementing Met to culture medium, we detected no methylthio-derivative of ansamitocin from A. pretiosum ATCC 31565 . This result suggests that only antibiotics with quinone moiety (such as benzoquinone and naphthoquinone ansamycins) are able to react chemically with methanethiol to form methylthio-derivatives.

Microorganisms such as Streptomyces can catabolize Met to methanethiol. Ashraf ${ }^{19}$ reported that L-methioninases, ubiquitous in all organisms except mammals, catalyzed the $\alpha, \gamma$-elimination of L-Met to $\alpha$-ketobutyrate, methanethiol and ammonia. A blast search of genome sequences of Streptomyces in NCBI indicated that L-methioninases existed in various species of Streptomyces including Streptomyces hygroscopicus (most of these L-methioninases were annotated as either methionine gamma-lyase or cystathionine gamma-synthases). When we added some Met to ISP2 medium to

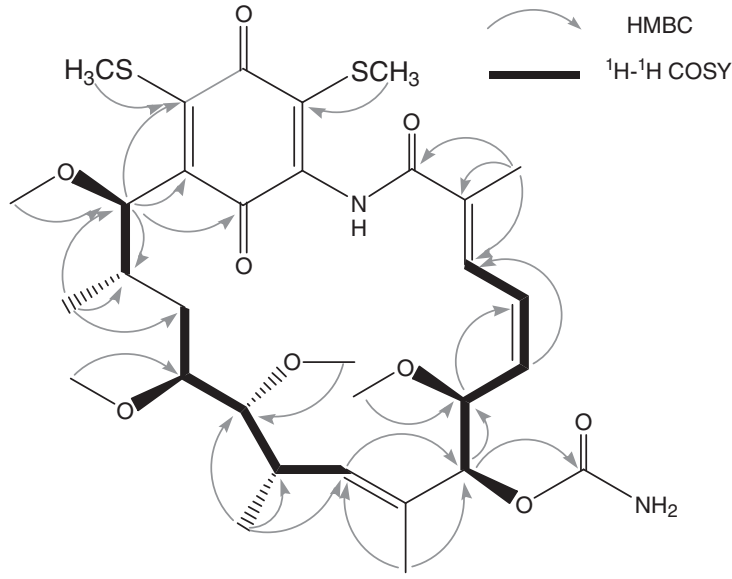

Figure 5 NMR correlations for 17,19-dimethylthioherbimycin A. A full color version of this figure is available at The Journal of Antibiotics journal online.

culture Streptomyces lividans TK24, a model streptomycete strain, we could smell the appearance of methanethiol (if GDM was also added, 1 could be detected by silica gel TLC).

S. hygroscopicus 17997 produced low level (about $3 \mathrm{mgl}^{-1}$ ) of $\mathbf{1}$ in a culture medium consisted of $2 \%$ starch, $0.5 \%$ glucose, $0.5 \%$ cottonseed meal, $1 \%$ cornsteep liquor, $0.5 \%$ yeast powder and $0.2 \%$ $\mathrm{CaCO}_{3}$. One possible explanation for low level production of 1 may be that this culture medium with rich organic nitrogen resources, could provide more sulfur-containing amino acids (Met and cysteine) than needed for normal growth of S. hygroscopicus 17997. Therefore, a part of the surplus Met was catabolized to methanethiol, which then reacted chemically with GDM to form $\mathbf{1}$.

Thiol-containing compounds such as ethanethiol, glutathione (GSH), $N$-acetyl-L-cysteine, and so on, can also react chemically with benzoquinone or naphthoquinone ansamycins, producing many semisynthetic or natural thioansamycins. ${ }^{12,20,21}$ Recently, Yang et al. ${ }^{22}$ reported two thionaphthomycins (naphthomycins $\mathrm{M}$ and $\mathrm{N})$. Naphthomycin $\mathrm{M}$ contains a thioglycolic acid group connected to $\mathrm{C}-30$ of the naphthomycin skeleton via sulfur, and naphthomycin $\mathrm{N}$ is a dimer-like molecule, with a 2 -aminoethanethiol group as bridge to connect two naphthomycin monomers. But the (bio)synthetic mechanism(s) of naphthomycins $\mathrm{M}$ and $\mathrm{N}$ remains unclear. ${ }^{22}$

Ōmura $^{23}$ and Shibata ${ }^{24}$ reported chemical modification of herbimycin $\mathrm{A}$, and obtained more than a dozen herbimycin A derivatives with various modifications at C-17 or C-19 of the benzoquinone moiety of herbimycin A. Among them, derivatives with a methylpiperazino group or bromine substituent at C-19 showed high antitumor activity. ${ }^{23,24}$ Our herbimycin A derivative 2 contains two identical substituents (methylthio-) at the benzoquinone moiety of herbimycin A, one at C-17 and the other at C-19 of the molecule. A preliminary cytotoxicity assay of 2 against HepG2 cancer cells was conducted by us using MTT assay. ${ }^{25}$ Compound 2 showed potent cytotoxic activity against HepG2 cancer cells, with an $\mathrm{IC}_{50}$ of $18.7 \mu \mathrm{M}$ (for herbimycin A, $14.0 \mu \mathrm{M}$ ), almost the same to 1 with an $\mathrm{IC}_{50}$ of $19.0 \mu \mathrm{M} .^{1}$

\section{ACKNOWLEDGEMENTS}

This work was supported by National Natural Science Foundation of China (81172964), National S\&T Major Special Project on Major New Drug Innovation (2012ZX09301-002-001 and 2010ZX09401-403), and Fundamental Research Funds for the Central Universities (2012N09). 
1 Liu, X. et al. A pair of sulfur-containing geldanamycin analogues, 19-S-methylgeldanamycin and 4,5-dihydro-19-S-methylgeldanamycin, from Streptomyces hygroscopicus 17997. J. Antibiot. 64, 519-522 (2011).

2 Hong, Y. S. et al. Inactivation of the carbamoyltransferase gene refines post-polyketide synthase modification steps in the biosynthesis of the antitumor agent geldanamycin. J. Am. Chem. Soc. 126, 11142-11143 (2004).

3 Rascher, A. et al. Cloning and characterization of a gene cluster for geldanamycin production in Streptomyces hygroscopicus NRRL 3602. FEMS Microbiol Lett 218, 223-230 (2003).

4 Shin, J. C. et al. Characterization of tailoring genes involved in the modification of geldanamycin polyketide in Streptomyces hygroscopicus JCM4427. J. Microbiol. Biotechnol. 18, 1101-1108 (2008).

5 Atta, M. et al. The methylthiolation reaction mediated by the Radical-SAM enzymes. Biochim. Biophys. Acta 1824, 1223-1230 (2012).

6 Futamura, Y. et al. Trierixin, a novel inhibitor of ER stress-induced XBP1 activation from Streptomyces sp. II. structure elucidation. J. Antibiot. 60, 582-585 (2007).

7 Funayama, S. et al. Structure of awamycin, a novel antitumor ansamycin antibiotic. J. Antibiot. 38, 1284-1286 (1985).

8 Celmer, W. D., Sciavolino, F. C., Cullen, W. P. \& Routien, J. B. 3-Methylthiorifamycins US Patent 3914218. Issued October 21, 1975.

9 Mochizuki, J. et al. New ansamycin antibiotics, naphthoquinomycins A and B, inhibitors of fatty acid synthesis in Escherichia coli. J. Antibiot. 39, 157-161 (1986).

10 Rohr, J. Biosynthetic formation of the S-methyl group of the angucycline antibiotic urdamycin E. J. Chem. Soc. Chem. Commun. 1989, 492-493 (1989).

11 Okabe, T. et al. Interaction of naphthomycin A with sulfhydryl compounds. J. Antibiot. 39, 316-317 (1986)

12 Sasaki, K. Novel geldanamycin derivative, its preparation, and drug comprising it as active ingredient Japan Patent 57-163369 A. (07-Oct-1982).

13 Ōmura, S. et al. Herbimycin, a new antibiotic produced by a strain of Streptomyces. J. Antibiot. 32, 255-261 (1979).
14 Ōmura, S., Nakagawa, A. \& Sadakane, N. Structure of herbimycin, a new ansamycin antibiotic. Tetrahedron Lett. 44, 4323-4326 (1979).

15 Lin, L. Z., Blaskó, G. \& Cordell, G. A. ${ }^{1} \mathrm{H}-\mathrm{NMR}$ analysis of herbimycins and dihydroherbimycins. J. Nat. Prod. 51, 1161-1165 (1988).

16 Kawamura, T., Tashiro, E., Yamamoto, K., Shindo, K. \& Imoto, M. SAR study of a novel triene-ansamycin group compound, quinotrierixin, and related compounds, as inhibitors of ER stress-induced XBP1 activation. J. Antibiot. 61, 303-311 (2008).

17 Sugita, M., Sasaki, T., Furihata, K., Seto, H. \& Otake, N. Studies on mycotrienin antibiotics, a novel class of ansamycins. II. Structure elucidation and biosynthesis of mycotrienins I and II. J. Antibiot. 35, 1467-1473 (1982).

$18 \mathrm{Yu}, \mathrm{T}$. W. et al. The biosynthetic gene cluster of the maytansinoid antitumor agent ansamitocin from Actinosynnema pretiosum. Proc. Natl Acad. Sci. USA 99, 7968-7973 (2002).

19 El-Sayed, A. M. Microbial L-methioninase: production, molecular characterization, and therapeutic applications. Appl. Microbiol. Biotechnol. 86, 445-467 (2010).

20 Cysyk, R. L. et al. Reaction of geldanamycin and C17-substituted analogues with glutathione: product identifications and pharmacological implications. Chem. Res. Toxicol. 19, 376-381 (2006).

21 Hooper, A. M. \& Rickards, R. W. 3-amino-5-hydroxybenzoic acid in antibiotic biosynthesis. XI. Biological origins and semisynthesis of thionaphthomycins, and the structures of naphthomycins I and J. J. Antibiot. 51, 845-851 (1998).

22 Yang, Y. H. et al. Naphthomycins L-N, ansamycin antibiotics from Streptomyces sp. CS. J. Nat. Prod. 75, 1409-1413 (2012).

23 Ōmura, S. et al. Chemical modification and antitumor activity of herbimycin A. 8,9Epoxide, 7,9-cyclic carbamate, and 17 or 19-amino derivatives. J. Antibiot. 37, 1264-1267 (1984).

24 Shibata, K. et al. Chemical modification of herbimycin A. Synthesis and in vivo antitumor activities of halogenated and other related derivatives of herbimycin A. J. Antibiot. 39, 415-423 (1986).

25 Mosmann, T. Rapid colorimetric assay for cellular growth and survival: application to proliferation and cytotoxicity assays. J. Immunol. Meth. 65, 55-63 (1983).

Supplementary Information accompanies the paper on The Journal of Antibiotics website (http://www.nature.com/ja) 